\title{
DGHO fordert verbesserte Bedingungen für die Durchführung nicht-kommerzieller Studien
}

\author{
Verleihung vier neuer Ehrenmitgliedschaften auf der \\ Gemeinsamen Jahrestagung der DGHO, ÖGHO und \\ SGMO
}

Auf der diesjährigen Gemeinsamen Jahrestagung der Deutschen, Österreichischen und Schweizerischen Fachgesellschaften für Hämatologie und Onkologie in Wien wurde aufgrund ihrer herausragenden Verdienste in der hämatologischen und onkologischen Therapieentwicklung am 11. Oktober folgenden vier Medizinern die Ehrenmitgliedschaft der DGHO Deutsche Gesellschaft Hämatologie und Onkologie e.V. verliehen:

Prof. Dr. med. Thomas Büchner (Münster)

Prof. Dr. med. Dr. h. c. Volker Diehl (Köln)

Prof. Dr. med. Rüdiger Hehlmann (Mannheim)

Prof. Dr. med. Dieter Hoelzer (Frankfurt)

Das Engagement der Geehrten in der Klinischen Forschung hat zur steten Verbesserung in Diagnose und Therapie bösartiger Bluterkrankungen geführt, deren Evidenz durch die erhöhten Heilungschancen der Patienten maßgeblich belegt ist. Diese Erfolge werden nicht nur durch die Verleihung der Ehrenmitgliedschaft gewürdigt, sondern auch als Anlass genommen, erneut bessere Studienbedingungen für nicht-kommerzielle Studien in Deutschland zu fordern. Die vor drei Jahren gesetzlich verabschiedete 12. Novelle des Arzneimittelgesetzes hat für die Durchführung nicht-kommerzieller klinischer Studien in Deutschland erhebliche Hürden geschaffen. Diese Studien fordern nunmehr einen vergleichbar hohen Verwaltungs- und Kostenaufwand wie die Zulassungsstudien neuer
Medikamente der pharmazeutischen Industrie. Dadurch sieht die DGHO die Innovationskraft der Forschung in Deutschland weiterhin gefährdet. Bislang wird die Studienlandschaft stark durch die Zulassungsstudien von Pharmaunternehmen dominiert. So stehen in Deutschland 179 nicht-kommerzielle klinische Studien 778 kommerziellen klinischen Studien gegenüber. Nach Meinung der Fachgesellschaft ist jedoch eine ausgeglichene Studienlandschaft dringend erforderlich. "Gerade in den nicht-kommerziellen klinischen Studien liegt ein großes Potential innovativer medizinischer Erkenntnisgewinnung», sagte Prof. Gerhard Ehninger, Geschäftsführender Vorsitzender der DGHO in seiner Ansprache. «Nur in diesen Studien können wir zum Beispiel zur Therapie seltener Erkrankungen arbeiten oder die Wirksamkeit zugelassener Arzneimittel direkt vergleichen, die nicht im unmittelbaren Herstellerinteresse liegen», so Ehninger weiter. Für seine Verdienste für die Deutsche Hodgkin Studiengruppe und für seine Arbeit im Kompetenznetz Maligne Lymphome wurde Prof. Volker Diehl in Wien geehrt. Er betonte die hohe Qualität nicht-kommerzieller klinischer Studien in Deutschland: «Wir haben in den letzten 30 Jahren bewiesen, dass der Studienpatient der am besten behandelte Patient ist». Auch Prof. Dieter Hoelzer, der für sein Engagement in der Leukämieforschung geehrt wurde, wies in seiner Ansprache darauf hin, dass die Studiengruppen bereits seit Beginn in eigener Initiative Qualitätssicherungsmaßnahmen für nichtkommerzielle klinische Studien entwickelten. Weiterhin akzentuierte er die Bedeutung nicht-kommerzieller Studien. «Im Bereich der Betreuung von Patienten mit Akuten Lymphatischen Leukä-

\section{KARGER}

Fax +497614520714

Information@Karger.de

www.karger.com (c) 2008 S. Karger GmbH, Freiburg 
mien behandeln wir heute 85 Prozent innerhalb von klinischen Studien».

Prof. Thomas Büchner sprach die unsichere Finanzierungslage für klinische Studien an. Der Wissenschaftler, der für seine Verdienste in der Biologie und Behandlung Akuter Myeloischer Leukämien geehrt wurde, betonte, dass «der Wegfall der Förderung klinischer Studien mit öffentlichen Geldern durch Institutionen wie die Deutsche Krebshilfe kaum aufgefangen werden kann». Für seine Verdienste im Kompetenznetz Leukämien wurde ebenfalls Prof. Rüdiger Hehlmann geehrt. «Deutsche klinische Studien in der Hämatologie galten bislang weltweit als beispielhaft», hob der Wissenschaftler anlässlich der Ehrung in Wien hervor. «Wenn die Finanzierung und Finanzierbarkeit nicht-kommerzieller Studien nicht sicher gestellt werden kann, droht eine Monopolisierung klinischer Forschung bei der Pharmaindustrie», so Hehlmann weiter.

Die DGHO sieht hier dringenden Handlungsbedarf und fordert, schnellstmöglich verbesserte Rahmenbedingungen in der nicht-kommerziellen klinischen Forschung zu schaffen. Dazu gehört einerseits entscheidend den Verwaltungsund Kostenaufwand zu mindern und andererseits das bisherige Versäumnis zur Beschaffung adäquater Mittel auszuräumen.

\section{Weitere Informationen}

Deutsche Gesellschaft für Hämatologie und Onkologie e.V.

DGHO Hauptstadtbüro

V.i.S.d.P. Mirjam Renz

Tel. +49 (0)30 / $27876089-0$

Fax +49 (0)30/ $27876089-18$

Mobil +49(0)160/ 7053015

renz@dgho.de

www.dgho.de 